\title{
OPEN The impact of bariatric surgery on serum tryptophan-kynurenine pathway metabolites
}

\author{
Kai Tai Derek Yeung ${ }^{1}$, Nicholas Penney ${ }^{1}$, Luke Whiley ${ }^{1,2,4}$, Hutan Ashrafian ${ }^{1}$, \\ Matthew R. Lewis ${ }^{3,4}$, Sanjay Purkayastha ${ }^{1}$, Ara Darzi ${ }^{1}$ \& Elaine Holmes ${ }^{1,2}$
}

This study aims to explore the immediate effects of bariatric surgery on serum tryptophan-kynurenine pathway metabolites in individuals with type 2 diabetes and $\mathrm{BMI}>30$. With the goal of providing insight into the link between tryptophan pathway metabolites, type 2 diabetes, and chronic obesityinduced inflammation. This longitudinal study included 20 participants. Half were diagnosed with type 2 diabetes. 11 and 9 underwent RYGB and SG respectively. Blood samples were obtained at pre-operative and 3 months post-operative timepoints. Tryptophan and downstream metabolites of the kynurenine pathway were quantified with an ultrahigh-performance liquid chromatography tandem mass spectrometry with electrospray ionisation method. At 3 months post-operation, RYGB led to significant reductions in tryptophan, kynurenic acid and xanthurenic acid levels when compared to baseline. Significant reductions of the same metabolites after surgery were also observed in individuals with T2D irrespective of surgical procedure. These metabolites were significantly correlated with serum HbA1c levels and BMI. Bariatric surgery, in particular RYGB reduces serum levels of tryptophan and its downstream kynurenine metabolites. These metabolites are associated with T2D and thought to be potentially mechanistic in the systemic processes of obesity induced inflammation leading to insulin resistance. Its reduction after surgery is associated with an improvement in glycaemic control (HbA1c).

Morbid obesity is a growing global epidemic associated with a number of significant health conditions leading to a chronic and increasingly heavy burden on healthcare systems. Co-morbidities include type 2 diabetes (T2D), hypertension, cardiovascular disease, non-alcoholic fatty liver disease, chronic kidney disease, certain cancers and infertility.

Bariatric surgery is one of the most effective long-term treatments for patients with morbid obesity and its associated co-morbidities ${ }^{1-3}$. It leads to significantly improved glycaemic control ${ }^{1,4}$, and reduced long term incidences of T2D-associated complications ${ }^{5}$. There are many postulated mechanisms of action, which contribute to such improvements, including calorie restriction, weight loss, modifications in bile acid metabolism, alteration in gut hormones and in the gut microbiome ${ }^{6}$. Some of these changes seem to occur in the early post-operative period and may be independent to weight loss ${ }^{7}$. The different types of bariatric surgery have different success rates with respect to weight loss, improvement of cardiovascular disease risk and remission of sleep apnoea with the Roux-En-Y gastric bypass (RYGB) achieving the most noteworthy results ${ }^{8,9}$. There is also currently conflicting evidence and debate as to whether certain types of operations favour T2D remission ${ }^{8,10-12}$.

Patients with obesity have an underlying chronic low-grade systemic inflammatory state. Pro-inflammatory factors are secreted by immune cells that accumulate in visceral adipose tissue ${ }^{13}$. Previously it has been reported that this systemic inflammation is reflected in an individual's metabolic phenotype ${ }^{14,15}$ with metabolites from the tryptophan (TRP)-kynurenine (KYN) pathway thought to be potentially mechanistic in the systemic processes that lead to clinical insulin resistance ${ }^{15}$.

TRP is an essential amino acid found in food sources such as milk, meat, fish, eggs and cocoa. Whilst TRP is critical for endogenous protein synthesis ${ }^{16}$, it is also catabolised into bioactive metabolites including kynurenines, serotonin and indoles, which are components of pathways that have a wide range of systemic effects on

\footnotetext{
${ }^{1}$ Department of Surgery \& Cancer, Imperial College London, South Kensington, London, UK. ${ }^{2}$ Australian National Phenome Centre \& Centre for Computational \& Systems Medicine, Health Futures Institute, Murdoch University, Perth, WA, Australia. ${ }^{3}$ National Phenome Centre, Imperial College London, South Kensington, London, UK. " Department of Metabolism, Digestion and Reproduction, Imperial College London, South Kensington, London, UK. ${ }^{\circledR}$ email: elaine.holmes@imperial.ac.uk
} 


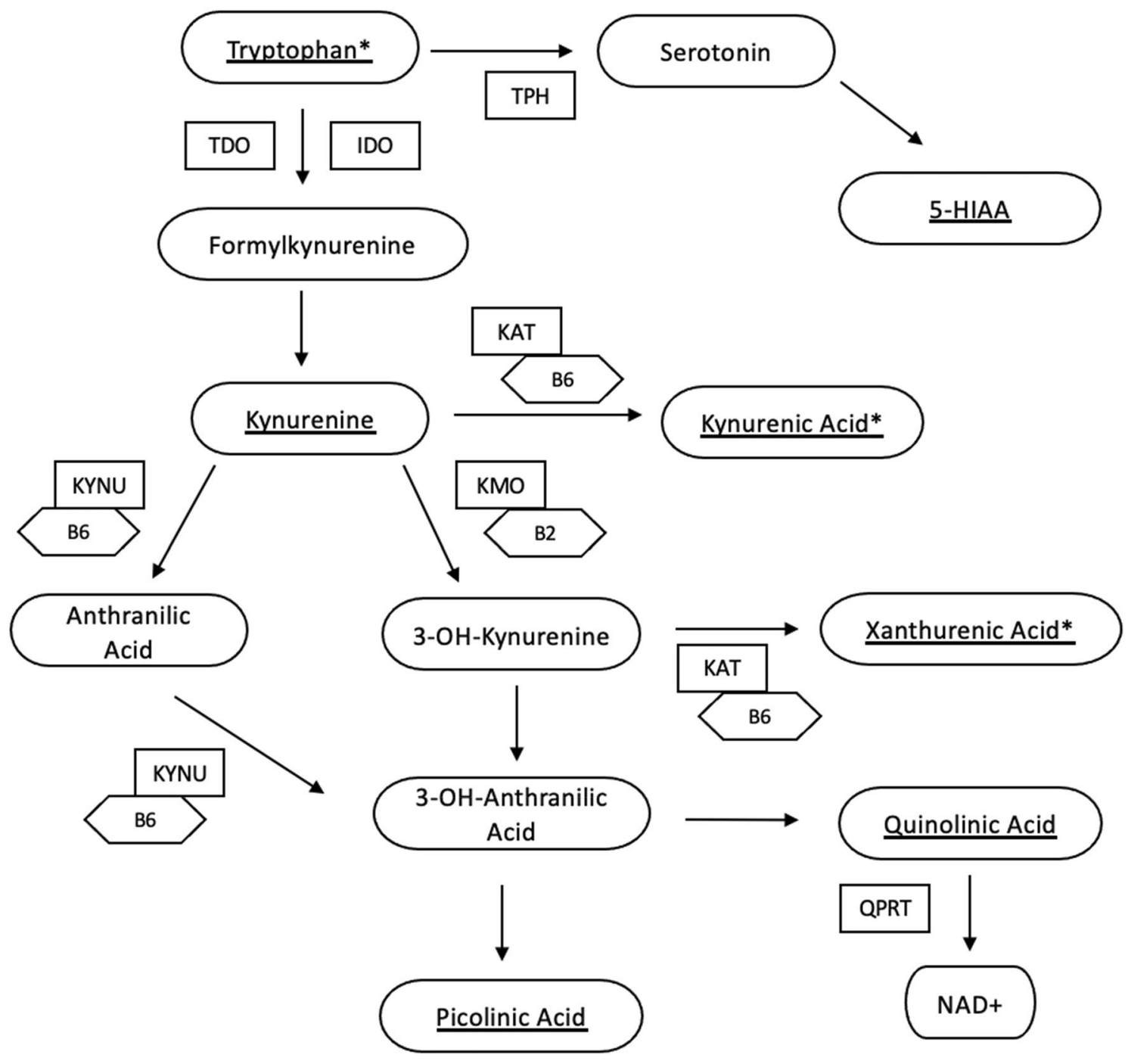

Figure 1. Tryptophan metabolism via kynurenine and serotonin metabolic routes. Underlined metabolites were quantified in this study. ${ }^{\star}$ Indicates key metabolites discussed in this study. Key: TPH tryptophan hydroxylase, TDO tryptophan 2,3-dioxygenase, IDO indoleamine 2,3-dioxygenase, KAT kynurenine aminotransaminase, KMO kynurenine 3 monooxygenase, KYNU kynureninase, Vit B6 pyridoxal 5-phosphate, Vit B2 Flavin Adenine Dinocleotide, 5-HIAA 5-Hydroxyindoleacetic acid, NAD nicotinamide adenine dinucleotide, QPRT quinolinate phosphoribosyltransferase.

the gut, brain, nervous system and microbiome as well as the regulation of systemic inflammation ${ }^{17}$. Most of the bioavailable tryptophan is oxidised and subsequently catabolised via the kynurenine pathway (Fig. 1). Further minor routes of catabolism include hydroxylation (serotonin), decarboxylation (tryptamine), and transamination (indolepyruvic acid) ${ }^{18}$.

The TRP-KYN pathway is of particular interest in obesity and has associations with the systemic inflammation that occurs in the condition. Driving this association is the first step in the pathway, TRP to KYN, which is catalysed by the enzymes tryptophan 2,3 dioxygenase (TDO) in the liver, or by indoleamine 2,3-dioxygenase (IDO) in extrahepatic tissues. IDO is preferentially induced by inflammatory cytokines such the Th1-type cytokine IFN $-\gamma^{19}$. In obesity, such cytokines are produced by pro-inflammatory macrophages found within adipose tissue ${ }^{13}$. Furthermore, the kynurenine tryptophan ratio (KTR) is used as a measure of IDO activation and, by extension, cellular immune activation ${ }^{20}$. A higher KTR is associated with insulin resistance and reported to be indicative of the resultant systemic inflammation ${ }^{21}$.

Further linking the pathway to obesity, KYN can then be hydroxylated to 3-hydroxy-L-kynurenine $(3 \mathrm{OH}-$ $\mathrm{KYN}$ ) by kynurenine 3-monooxygenase (KMO), which is present in macrophages within adipose tissue but not primary adipocytes. $3 \mathrm{OH}-\mathrm{KYN}$ is subsequently metabolised to either, xanthurenic acid (XA), or 3 hydroxyanthranilic acid. A shift towards overproduction of XA within this pathway has been associated with the impairment of production, release and the consequent metabolic effects of insulin ${ }^{21}$.

In summary, obesity leads to chronic systemic inflammation. Metabolites from the TRP-KYN pathway are potentially mechanistic in this process as well as in the development of obesity related co-morbidities such as T2D. 
Bariatric surgery leads to rapid and sustained resolution of obesity and its related co-morbidities. This study aims to utilise these powerful metabolic effects to provide an insight into the link between tryptophan-kynurenine pathway metabolites, T2D, and obesity induced systemic inflammation. Its implications include identifying mechanisms of action that lead to such beneficial metabolic benefits and potentially the identification of future surgical or pharmalogical therapeutic targets.

\section{Study design and methods}

Sample procurement and spectrometric analysis. Study participants were recruited prospectively between July 2015 to December 2016 and fulfilled criteria for bariatric surgery in line with National Institute for Health and Care Excellence guidance ${ }^{22}$. All individuals underwent local multidisciplinary team review and approval prior to proceeding to undergo Roux-En-Y Gastric Bypass (RYGB) or Vertical Sleeve Gastrectomy (VSG) surgery at St Mary's Hospital, London, United Kingdom between August 2015 and July 2018. Specific inclusion and exclusion criteria are listed in Supplement 1.

Clinical parameters were measured, and blood samples were drawn as part of the routine pre- and 3-month post-operative outpatient clinical review appointments. Blood was collected using additive free red top BD Vacutainer ${ }^{\circledast}$ (New Jersey, USA) tubes. After $45 \mathrm{~min}$ at room temperature, the tubes were centrifuged at $12,000 \times g$ for $15 \mathrm{~min}$. The resultant serum supernatant was aliquoted and stored at $-80^{\circ} \mathrm{C}$ until analysis.

TRP and the downstream metabolites of the KYN pathway were quantified for all samples and a series of pooled QC samples, using an established targeted ultrahigh-performance liquid chromatography tandem mass spectrometry method utilising electrospray ionisation (UHPLC-ESI-MS/MS) ${ }^{23}$. In addition to direct TRP metabolites, neopterin was also quantified as a marker of cellular inflammation as it is produced by IFN- $\gamma$ stimulated macrophages. Citrulline was measured as a marker of gut health related to enterocyte mass reduction ${ }^{24}$. Indole-3-acetic acid (IAA) is a gut microbiota-derived metabolite from dietary $\mathrm{TRP}^{25}$ and was measured as a marker of microbiome influence on the indole pathway.

Statistical analysis. Following acquisition, mass spectrometry data were integrated using an established protocol described by Whiley et al. that employed quantification calibration and appropriate quality control checks ${ }^{23}$ which resulted in a concentration value for each metabolite. Concentration data points that were outside of three standard deviations from the mean were considered outliers and excluded from analysis.

The Shapiro Wilk test was performed on all measured metabolites to determine normality. The majority of the measured metabolites were not normally distributed (see Supplement 2). Significant differences in tryptophan pathway metabolite abundances between surgical intervention and diabetes subgroups were established using the non-parametric Mann-Whitney U test. To control for false discovery rate (FDR), q values were generated using the method described by Benjamini and Hochberg $(\mathrm{BH})^{26}$. A q value FDR threshold of $<0.05$ was employed throughout the study. Repeated measures correlation (rmcorr) ${ }^{27-29}$ was employed to measure the common regression slope of paired measures for multiple individuals. The calculated repeated measures coefficient $\left(r_{r m}\right)$ is interpreted in the same manner as a correlation coefficient with a value of 1 suggested of data with perfect paired association and a value of -1 suggestive of a perfect negative association. The difference $\Delta$ (3 months-baseline) of measured metabolites was also evaluated for each participant to further investigate the effect of bariatric surgery on these metabolites. Correlations between variables was assessed using Spearman's Rank correlation. All statistical analysis and graphical representations were conducted and produced with R ( $\mathrm{v}$ 4.0.1) run in R Studio (v1.3.959).

Study approval and informed consent. The study was approved by East of Scotland Research Ethics Committee (15/ES/0026). Joint Research Compliance Reference Number 15SM2479 and IRAS project ID 169767.

All procedures performed in studies involving human participants were in accordance with the ethical standards of the institutional and/or national research committee and with the 1964 Helsinki declaration and its later amendments or comparable ethical standards. Informed consent was obtained from all individual participants included in the study.

\section{Results}

Study baseline characteristics. Of the 20 patients included in the study (mean age and BMI of 47.05 and 49 respectively), 11 and 9 patients underwent RYGB and SG respectively. Half the patients had T2D. The overall baseline demographics prior to subgroup division are displayed in Table 1.

The study cohort was then divided into the following four subgroups: individuals with and without T2D prior to surgery, and those stratified by their surgical intervention (RYGB or VSG) (Tables 2, 3). Separate sets of analysis were completed to compare the impact of bariatric surgery on the TRP-KYN pathway in participants with T2D vs Non-Diabetes (ND) and RYGB vs VSG.

Pre-operative and 3-month post-operative metabolite comparisons-surgical intervention. Both surgical interventions led to significant reductions in weight across all subgroups at 3 months post-operation (Table 2). HbA1c concentrations were significantly reduced at the 3-month timepoint following RYGB, but not within the VSG group (Table 2).

RYGB intervention resulted in a significant decrease in kynurenic acid (KYNA) ( $p=0.010, \mathrm{BH} q=0.048)$, $\mathrm{XA}(\mathrm{p}=0.001, \mathrm{BH} q=0.017)$ and TRP $(\mathrm{p}=0.002, \mathrm{BH} \mathrm{q}=0.017)$ concentrations (Fig. 2). Concentrations of $3-\mathrm{OH}$ kynurenine $(\mathrm{p}=0.023, \mathrm{BH} \mathrm{q}=0.082)$ and quinolinic acid $(\mathrm{p}=0.043, \mathrm{BH} q=0.121)$ also decreased, however presented $\mathrm{BH}$ q values of $>0.05$ post control of the FDR. The VSG procedure did not result in any significant changes 


\begin{tabular}{|l|l|}
\hline \multicolumn{2}{|l|}{ Baseline demographics } \\
\hline $\mathrm{n}$ & 20 \\
\hline Sex, $\mathrm{n}(\mathrm{M}: \mathrm{F})$ & $20(5: 15)$ \\
\hline Age (years) & $47.05 \pm 9.23$ \\
\hline Weight $(\mathrm{kg})$ & $131.00 \pm 21.34$ \\
\hline BMI $\left(\mathrm{kg} / \mathrm{m}^{2}\right)$ & $49.00 \pm 7.48$ \\
\hline T2D, $\mathrm{n}(\%)$ & $10(50)$ \\
\hline HbAlc $(\mathrm{mmols} / \mathrm{mol})$ & $51.35 \pm 18.18$ \\
\hline Metformin, $\mathrm{n}(\%)$ & $9(45)$ \\
\hline Insulin, $\mathrm{n}(\%)$ & $2(5)$ \\
\hline
\end{tabular}

Table.1. Baseline demographics. All listed data are means \pm standard deviation unless stated otherwise.

\begin{tabular}{|l|l|l|l|l|}
\hline \multirow{2}{*}{ Subgroups } & \multicolumn{2}{|l|}{ RYGB } & VSG \\
\cline { 2 - 5 } & Pre Op & Post Op & Pre Op & Post Op \\
\hline $\mathrm{n}$ & 11 & & 9 & \\
\hline Sex, $\mathrm{n}(\mathrm{M}: \mathrm{F})$ & $1: 10$ & & $4: 5$ & \\
\hline Age (years) & $48.27 \pm 9.65$ & & $45.56 \pm 9.00$ & \\
\hline Weight $(\mathrm{kg})$ & $124.73 \pm 18.80$ & $98.03 \pm 14.04^{*}$ & $138.67 \pm 22.78$ & $114.80 \pm 20.63^{\star}$ \\
\hline BMI $\left(\mathrm{kg} / \mathrm{m}^{2}\right)$ & $48.32 \pm 6.12$ & $38.03 \pm 4.88^{\star}$ & $49.81 \pm 9.20$ & $41.19 \pm 7.99$ \\
\hline T2D, $\mathrm{n}(\%)$ & $7(64)$ & & $3(33)$ & \\
\hline Metformin, $\mathrm{n}(\%)$ & $6(55)$ & $4(36)$ & $3(33)$ & $3(33)$ \\
\hline Insulin, $\mathrm{n}(\%)$ & $2(18)$ & $1(9)$ & $0(0)$ & $0(0)$ \\
\hline HbAlc $(\mathrm{mmols} / \mathrm{mol})$ & $51 \pm 17.78$ & $38.09 \pm 7.73^{\star}$ & $51.78 \pm 19.75$ & $39.00 \pm 7.52$ \\
\hline
\end{tabular}

Table.2. Effect of surgery by surgical procedure subgroups. ${ }^{*} \mathrm{p}<0.05$ pre- and post-op comparison.

\begin{tabular}{|l|l|l|l|l|}
\hline \multirow{2}{*}{ Subgroups } & \multicolumn{2}{|l|}{ T2D } & Non diabetes \\
\cline { 2 - 5 } & Pre Op & Post Op & Pre Op & Post Op \\
\hline $\mathrm{n}$ & 10 & & 10 & \\
\hline Sex, n (M:F) & $4: 6$ & & $1: 9$ & \\
\hline Age (years) & $51.7 \pm 6.29$ & & $42.20 \pm 9.59$ & \\
\hline Weight (kg) & $130.40 \pm 22.00$ & $104.06 \pm 17.45^{*}$ & $131.60 \pm 21.83$ & $107.06 \pm 21.02^{\star}$ \\
\hline BMI (kg/m $)$ & $48.14 \pm 7.60$ & $38.43 \pm 5.97^{*}$ & $49.85 \pm 7.66$ & $40.48 \pm 7.12^{*}$ \\
\hline Metformin, n (\%) & $9(90)$ & $7(70)$ & $0(0)$ & $0(0)$ \\
\hline Insulin, n (\%) & $2(20)$ & $1(10)$ & $0(0)$ & $0(0)$ \\
\hline HbAlc (mmols/mol) & $65.10 \pm 16.41^{+}$ & $43.20 \pm 7.57^{*}$ & $37.60 \pm 2.95$ & $33.80 \pm 3.26^{*}$ \\
\hline RYGB, n (\%) & $7(70)$ & & $4(40)$ & \\
\hline VSG, n (\%) & $3(30)$ & & $6(60)$ & \\
\hline
\end{tabular}

Table.3. Effect of surgery by T2D diagnosis subgroups. ${ }^{+}$Significant difference between baseline demographics. ${ }^{*} \mathrm{p}<0.05$ pre- and post-op comparison.

in metabolite concentrations at the 3-month timepoint (Table 4) but post-operative trend changes mirrored those observed in the RYGB group. No significant difference was found in the kynurenine/tryptophan ratio for either the RGYB or VSG groups after surgery.

Pre-operative and 3-month post-operative metabolite comparisons-T2D vs non-diabetes. At pre-operative baseline, there was a significantly higher concentration of HbAlc in those participants with T2D, which was expected. In addition, serum tryptophan $(\mathrm{p}=0.005)$ and xanthurenic acid $(\mathrm{p}=0.015)$ concentrations were higher in those with T2D (Fig. 3), although after controlling for the FDR, BH q values for both metabolites were $>0.05(\mathrm{q}=0.10$ and $\mathrm{q}=0.14$ respectively).

Following surgical intervention, HbA1c concentrations were significantly reduced in both the T2D and Non-Diabetes (ND) groups, with a greater reduction observed in the T2D group. In the T2D group, significant reductions in the concertation of XA $(\mathrm{p}=0.004, \mathrm{BH} \mathrm{q}=0.030)$ and $\mathrm{TRP}(\mathrm{p}=0.003, \mathrm{BH} \mathrm{q}=0.030)$ occurred 

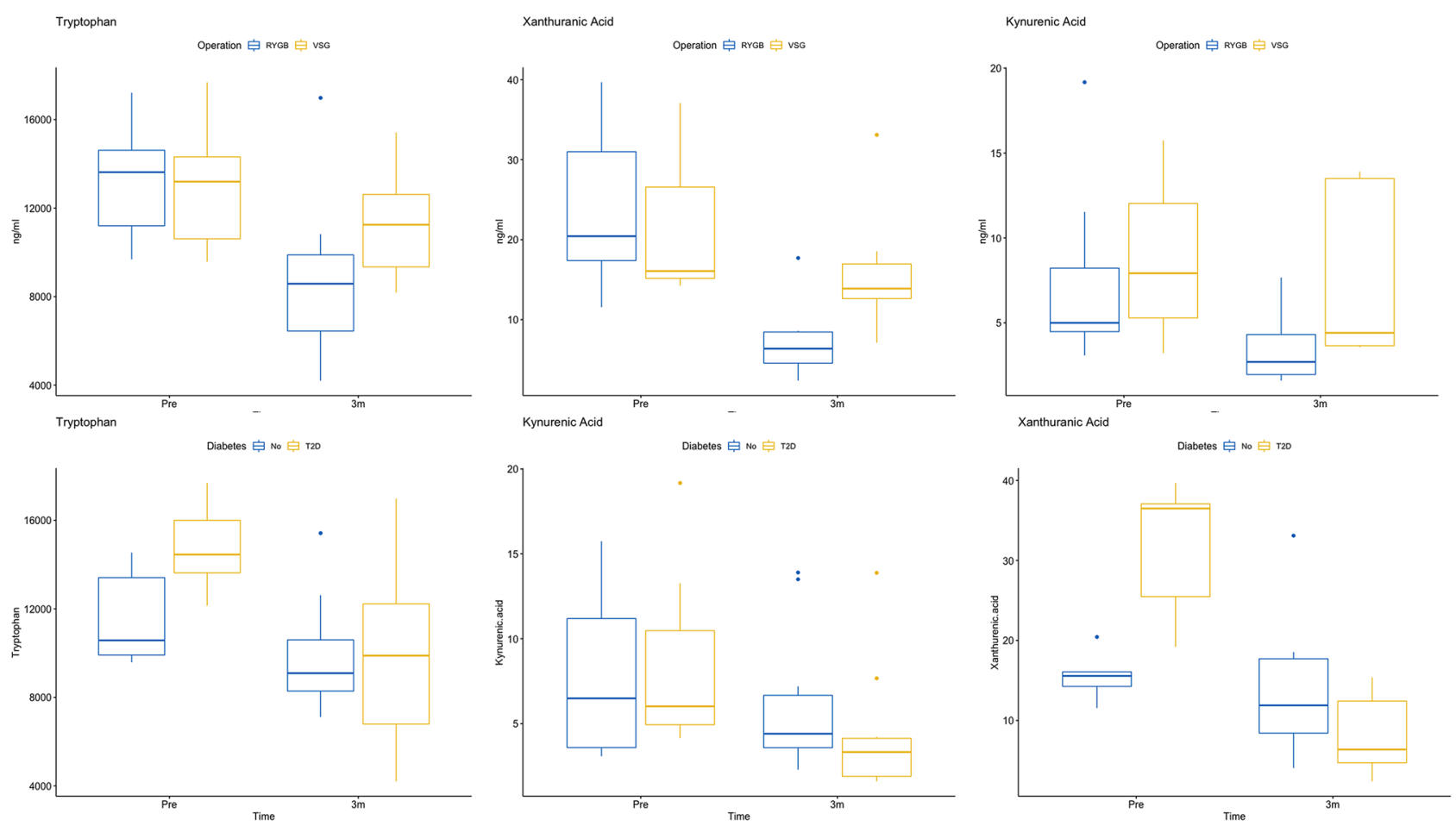

Figure 2. Pre- and post operative concentrations of tryptophan, kynurenic acid and xanthurenic acid shown with surgical procedure (top row) and T2D subgroups (bottom row).

\begin{tabular}{|c|c|c|c|c|c|c|c|c|}
\hline & \multicolumn{4}{|l|}{ RYGB } & \multicolumn{4}{|l|}{ VSG } \\
\hline & Pre Op & Post Op & p value & q value & Pre Op & Post Op & p value & q value \\
\hline Picolinic acid & $7.39 \pm 3.93$ & $5.98 \pm 1.51$ & 0.933 & 0.933 & $5.32 \pm 1.28$ & $6.58 \pm 1.73$ & 0.181 & 0.699 \\
\hline $\begin{array}{l}3 \text { Hydroxy- } \\
\text { anthranilic } \\
\text { acid }\end{array}$ & $15.67 \pm 8.77$ & $14.79 \pm 0.59$ & 0.533 & 0.747 & $15.31 \pm 3.1$ & $13.32 \pm \mathrm{NA}$ & 0.857 & 0.923 \\
\hline $\begin{array}{l}\text { Quinolinic } \\
\text { acid }\end{array}$ & $67.49 \pm 15.35$ & $53.95 \pm 24.84$ & 0.043 & 0.121 & $68.44 \pm 20.14$ & $64.55 \pm 17.87$ & 0.605 & 0.804 \\
\hline Citrulline & $3548.68 \pm 1608.63$ & $2790.11 \pm 840.02$ & 0.217 & 0.434 & $4264.1 \pm 1337.04$ & $3296.14 \pm 880.96$ & 0.113 & 0.699 \\
\hline $\begin{array}{l}\text { Indole } 3 \\
\text { acetic acid }\end{array}$ & $319.93 \pm 269.57$ & $425.13 \pm 350.29$ & 0.401 & 0.624 & $264.97 \pm 154.65$ & $361.19 \pm 268.67$ & 0.666 & 0.804 \\
\hline $\begin{array}{l}\text { Kynurenic } \\
\text { acid }\end{array}$ & $7.06 \pm 4.75$ & $3.34 \pm 1.87$ & 0.010 & 0.048 & $8.89 \pm 4.48$ & $7.5 \pm 4.83$ & 0.596 & 0.804 \\
\hline $\begin{array}{l}\text { 5-Hydrox- } \\
\text { yindole acetic } \\
\text { acid }\end{array}$ & $9.53 \pm 5.06$ & $8.33 \pm 2.49$ & 0.847 & 0.912 & $8.09 \pm 3.82$ & $6.36 \pm 1.49$ & 0.340 & 0.794 \\
\hline $\begin{array}{l}\text { Xanthurenic } \\
\text { acid }\end{array}$ & $24.06 \pm 10.53$ & $7.33 \pm 4.78$ & 0.001 & 0.017 & $22.47 \pm 12.68$ & $16.19 \pm 8.23$ & 0.267 & 0.747 \\
\hline Kynurenine & $317.55 \pm 69.72$ & $248.94 \pm 77.3$ & 0.065 & 0.152 & $317.64 \pm 73.11$ & $297.82 \pm 65.44$ & 0.606 & 0.804 \\
\hline $\begin{array}{l}30 \mathrm{H} \text { Kynure- } \\
\text { nine }\end{array}$ & $10.56 \pm 4.35$ & $6.81 \pm 2.65$ & 0.023 & 0.082 & $10.13 \pm 3.13$ & $7.35 \pm 2.03$ & 0.063 & 0.699 \\
\hline Neopterin & $2.67 \pm 1.59$ & $2.91 \pm 1.93$ & 0.844 & 0.912 & $2.35 \pm 0.94$ & $2.44 \pm 0.65$ & 0.489 & 0.804 \\
\hline Tryptophan & $13,242.5 \pm 2443.76$ & $8711.05 \pm 3482.81$ & 0.002 & 0.017 & $12,892.19 \pm 2741.4$ & $11,291.87 \pm 2413.88$ & 0.200 & 0.699 \\
\hline $\begin{array}{l}\text { Kynurenine/ } \\
\text { tryptophan } \\
\text { ratio }\end{array}$ & $0.025 \pm 0.006$ & $0.026 \pm 0.007$ & 0.842 & 0.912 & $0.024 \pm 0.004$ & $0.026 \pm 0.004$ & 0.689 & 0.804 \\
\hline
\end{tabular}

Table.4. Effect of surgery by surgical procedure subgroup on measured metabolites. q values were generated using the Benjamini and Hochberg method to control the false discovery rate (FDR). Units of all of metabolites displayed $-\mathrm{ng} / \mathrm{ml}$. KTR expressed as a ratio. Significant values are indicated by bold type. 
Tryptophan

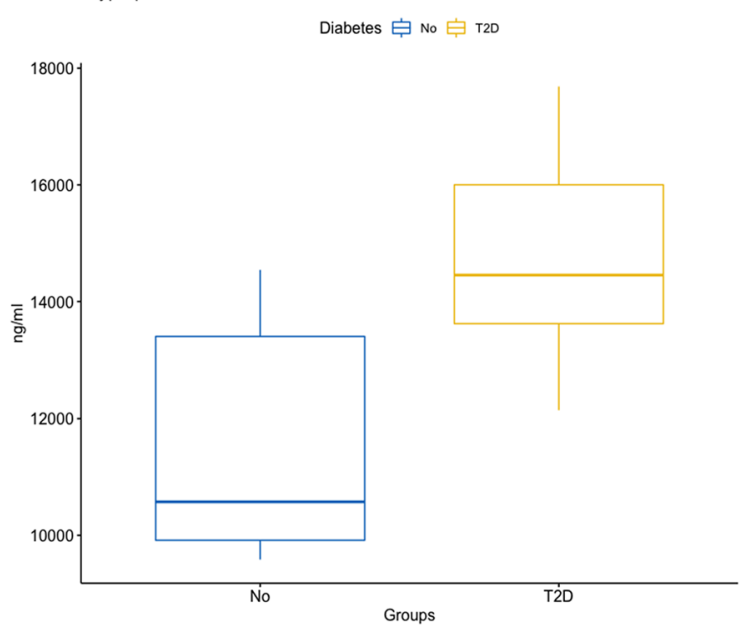

Xanthurenic acid

Diabetes 白 No 白 T2D

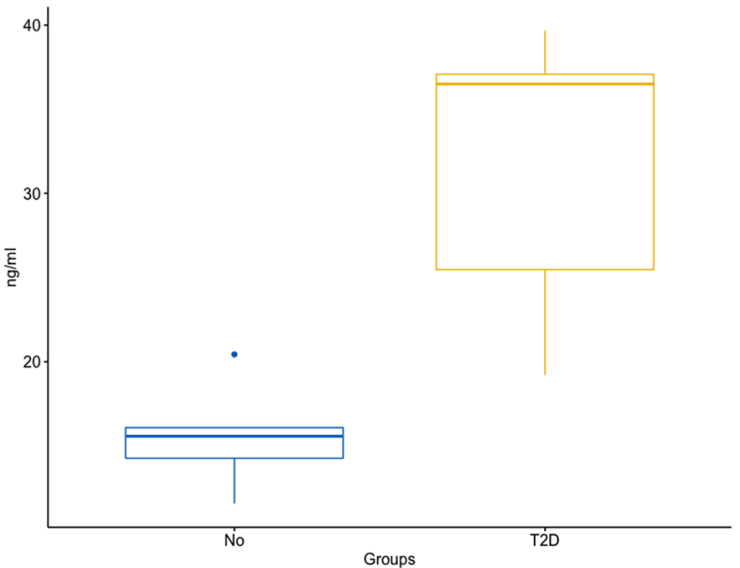

Figure 3. Baseline pre-operative concentrations of tryptophan and xanthurenic acid.

\begin{tabular}{|c|c|c|c|c|c|c|c|c|}
\hline & \multicolumn{4}{|l|}{ T2D } & \multicolumn{4}{|l|}{ ND } \\
\hline & Pre Op & Post Op & p value & q value & Pre Op & Post Op & $p$ value & q value \\
\hline Picolinic acid & $7.42 \pm 3.82$ & $5.83 \pm 1.08$ & 0.683 & 0.796 & $5.29 \pm 1.56$ & $6.68 \pm 1.86$ & 0.100 & 0.325 \\
\hline $\begin{array}{l}3 \text { Hydroxyan- } \\
\text { thranilic acid }\end{array}$ & $16.19 \pm 7.56$ & $14.3 \pm 0.94$ & 0.937 & 0.937 & $13.85 \pm 4.39$ & $\mathrm{NA} \pm \mathrm{NA}$ & 1.000 & 1.000 \\
\hline $\begin{array}{l}\text { Quinolinic } \\
\text { acid }\end{array}$ & $66.57 \pm 11.77$ & $58.16 \pm 25.84$ & 0.113 & 0.318 & $69.18 \pm 21.68$ & $59.7 \pm 19.11$ & 0.353 & 0.675 \\
\hline Citrulline & $3479.83 \pm 1516.31$ & $2950.59 \pm 918.29$ & 0.353 & 0.634 & $4261.41 \pm 1451.63$ & $3085.06 \pm 872.6$ & 0.063 & 0.301 \\
\hline $\begin{array}{l}\text { Indole } 3 \\
\text { acetic acid }\end{array}$ & $318.45 \pm 276.86$ & $350.77 \pm 238.45$ & 0.579 & 0.737 & $271.94 \pm 160.66$ & $441.95 \pm 375.87$ & 0.529 & 0.820 \\
\hline $\begin{array}{l}\text { Kynurenic } \\
\text { Acid }\end{array}$ & $8.22 \pm 4.91$ & $4.38 \pm 3.79$ & 0.011 & 0.054 & $7.54 \pm 4.51$ & $6.04 \pm 4.27$ & 0.579 & 0.820 \\
\hline $\begin{array}{l}\text { 5-Hydrox- } \\
\text { yindole acetic } \\
\text { acid }\end{array}$ & $9.98 \pm 4.92$ & $8.28 \pm 2.32$ & 0.571 & 0.737 & $7.78 \pm 3.95$ & $6.62 \pm 2.02$ & 0.631 & 0.820 \\
\hline $\begin{array}{l}\text { Xanthurenic } \\
\text { acid }\end{array}$ & $31.59 \pm 8.81$ & $8.19 \pm 5.33$ & 0.004 & 0.030 & $15.58 \pm 3.23$ & $13.65 \pm 8.73$ & 0.364 & 0.675 \\
\hline Kynurenine & $330.23 \pm 49.48$ & $282.51 \pm 79.95$ & 0.370 & 0.634 & $304.95 \pm 85.57$ & $262.71 \pm 71.78$ & 0.274 & 0.675 \\
\hline $\begin{array}{l}\text { 30H kynure- } \\
\text { nine }\end{array}$ & $11 \pm 3.98$ & $7.33 \pm 2.85$ & 0.029 & 0.101 & $9.74 \pm 3.63$ & $6.78 \pm 1.83$ & 0.070 & 0.301 \\
\hline Neopterin & $2.61 \pm 1.72$ & $3.03 \pm 2.01$ & 0.739 & 0.796 & $2.45 \pm 0.84$ & $2.37 \pm 0.57$ & 0.796 & 0.862 \\
\hline Tryptophan & $14,794.55 \pm 1848.93$ & $9922.06 \pm 3971.6$ & 0.003 & 0.030 & $11,565.41 \pm 2004.27$ & $9822.78 \pm 2557.61$ & 0.052 & 0.301 \\
\hline $\begin{array}{l}\text { Kynurenine/ } \\
\text { tryptophan } \\
\text { ratio }\end{array}$ & $0.023 \pm 0.004$ & $0.026 \pm 0.006$ & 0.408 & 0.634 & $0.027 \pm 0.006$ & $0.027 \pm 0.006$ & 0.762 & 0.862 \\
\hline
\end{tabular}

Table.5. Effect of surgery by T2D subgroup on measured metabolites. q values were generated using the Benjamini and Hochberg method to control the false discovery rate (FDR). Units of all of metabolites displayed $-\mathrm{ng} / \mathrm{ml}$. KTR expressed as a ratio. Significant values are indicated by bold type.

(Fig. 2). No significant metabolite changes were observed in the ND group at the 3-month post-surgery timepoint (Table 5). The kynurenine/tryptophan ratio did not significantly change between pre- and post-surgery for either the T2D or the ND group.

Metabolite relationship with HbA1c. When evaluated with repeated measures correlation, tryptophan $\left(r_{r m}=0.798, p=1.4 \mathrm{e}-5\right)$, kynurenic acid $\left(r_{r m}=0.629, p=0.002\right)$ and xanthurenic acid $\left(r_{r m}=0.619, p=0.006\right)$ were found to be positively associated with corresponding serum HbAlc concentrations at the time of sampling (Fig. 2). For $r_{r m}$ values of all measured metabolites with HbAlc please refer to Supplementary 3.

Metabolite relationship with BMI. Similarly, when evaluated with repeated measures correlation, tryptophan $\left(r_{r m}=0.670, p=9 e-4\right)$, kynurenic acid $\left(r_{r m}=0.507, p=0.018\right)$ and xanthurenic acid $\left(r_{r m}=0.693, p=0.0014\right)$ were found to be positively associated with corresponding BMI measurement at the time of sampling (Fig. 4). For $\mathrm{r}_{\mathrm{rm}}$ values of all measured metabolites with BMI please refer to Supplementary 4 . 

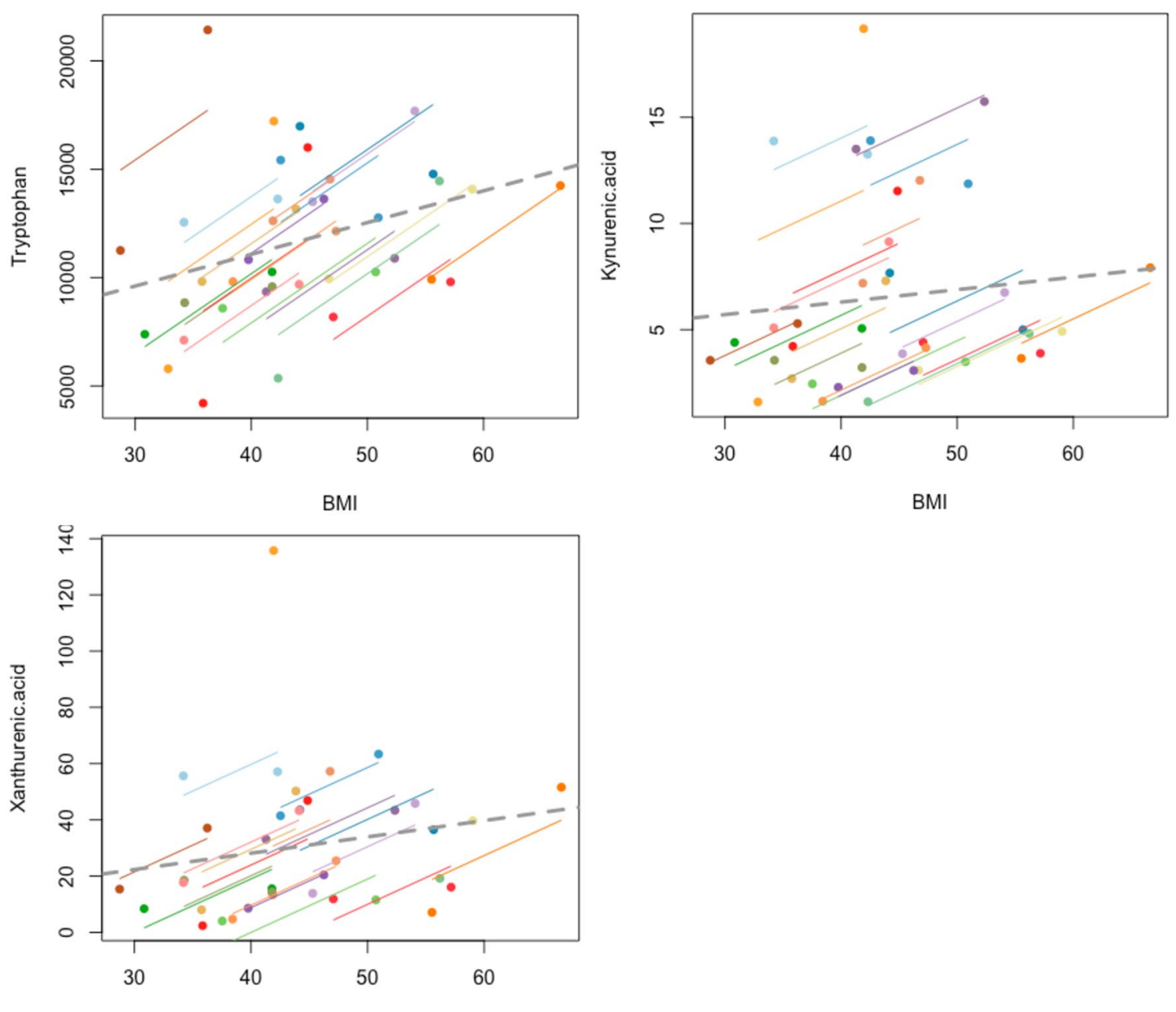

$\mathrm{BMI}$

Figure 4. Repeated measure correlation plots of BMI with tryptophan, kynurenic acid and xanthurenic acid levels respectively. Metabolite units $(\mathrm{ng} / \mathrm{ml}), \mathrm{BMI}\left(\mathrm{kg} / \mathrm{m}^{2}\right)$.

To further examine the effect of surgery on the KYN pathway, the correlation coefficient for the change $(\Delta)$ in concentration for each participant for each pathway metabolite with $\Delta$ BMI and $\Delta \mathrm{HbA} 1 \mathrm{c}$ was calculated (Fig. 5). Significant positive correlation was observed between $\Delta$ Hbalc and $\Delta$ Tryptophan $(r=0.57, p=0.003)$ and significant negative correlation between $\Delta \mathrm{Hba1c}$ and $\Delta \mathrm{KTR}(\mathrm{r}=-0.61, \mathrm{p}=0.004)$.

Changes in BMI were not found to be significantly correlated with any of the $\Delta$ of measured metabolites. Within the metabolic pathway, $\Delta$ Tryptophan showed a positive correlation with the downstream metabolites of $\Delta$ Kynurenic Acid $(\mathrm{R}=0.73, \mathrm{p}=0.0002), \Delta 5$-hydroxyindole acetic acid $(\mathrm{R}=0.54 \mathrm{p}=0.013)$ and $\Delta$ Xanthurenic Acid $(\mathrm{R}=0.68, \mathrm{p}=0.002)$.

\section{Discussion}

Roux-En-Y gastric bypass (RYGB) but not vertical sleeve gastrectomy (VSG) led to significant decreases in serum tryptophan (TRP), kynurenic acid (KYNA) and xanthurenic acid (XA) levels. In those participants with T2D, surgery led to significant decreases in HbAlc and in serum TRP and XA levels. TRP, KYNA and XA levels were found to be strongly correlated with the corresponding serum HbA1c concentrations and BMI at the time of sampling when assessed using repeated measures correlation. Finally, $\Delta$ in serum TRP levels were found to be significantly and positively correlated with $\triangle \mathrm{Hba} 1 \mathrm{C}$ and significantly and negatively correlated with $\Delta$ Kynurenine : Tryptophan Ratio (KTR). $\Delta$ BMI was not significantly correlated with any of the metabolites measured in this study.

Obesity induced inflammation and immunomodulation ${ }^{30}$ are believed to be one of the links between T2D and the tryptophan pathway ${ }^{17}$. Obesity is associated with increased gene expression of all key enzymes in the tryptophan-kynurenine pathway including IDO, KMO, kynureninase (KYNU) and kynurenine aminotransaminase (KAT) within adipose tissue and proinflammatory macrophages ${ }^{31}$. In particular, KMO activation may cause diversion of the pathway towards XA production. Our data support this with higher concentrations of XA reported in the pre-operative groups where BMI scores and obesity levels were greater, compared with the 3-month post-operative samples. 


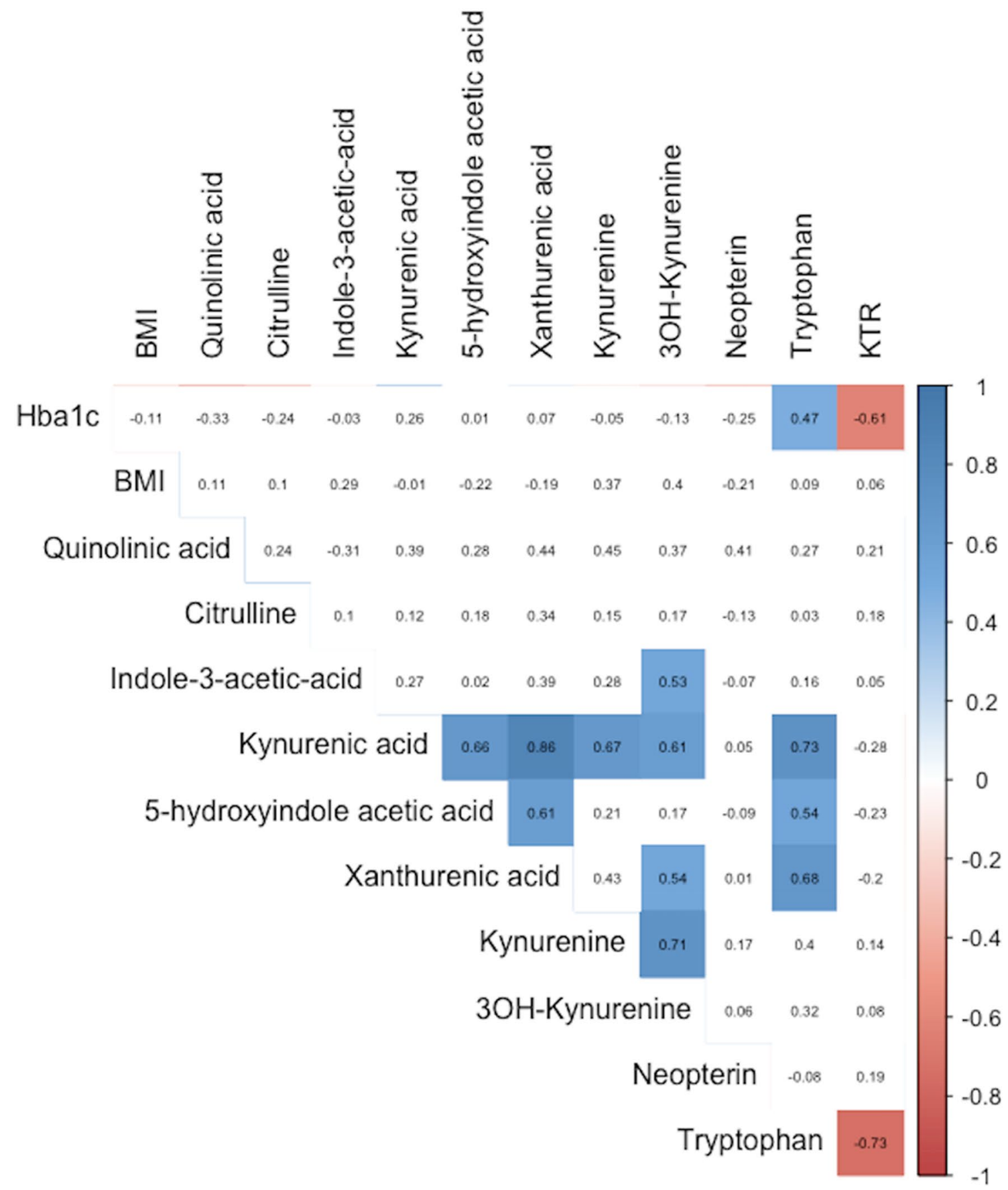

Figure 5. Heatmap displaying Spearman's Rank correlation co-efficient between $\Delta$ of measured parameters and pathway metabolites. Coloured squares indicate significant results $\mathrm{p}$ value of $<0.05$.

KMO expression has been reported to be positively associated with $\mathrm{HbAlc}$ levels ${ }^{31}$ suggesting that there may be an association between its activity and clinical T2D. In addition, XA is considered to be diabetogenic with previous studies reporting raised levels of XA in the urine $\mathrm{e}^{32,33}$ and serum ${ }^{34}$ in T2D. In relation to bariatric surgery, a reduction in XA has been reported with improved glucose control a year after surgery ${ }^{35}$. Our data are in agreement: we observed higher baseline XA concentrations in the T2D group compared to the ND group. In the T2D group, XA concentrations were dramatically reduced at the 3-month, post-operative timepoint, whilst concentrations in the ND group remained stable throughout the study. Interestingly, only surgical intervention with RYGB led to a significant reduction of XA at 3-months, whilst there were no significant changes in XA concentrations following the VSG procedure. XA also significantly correlated with HbA1c and BMI (Figs. 4, 6), again underscoring the association between XA and T2D. Although this observation does not demonstrate causality, the significant relationship supports the postulated contributory role of TRP-KYN pathway dysregulation in the pathogenesis of T2D. 

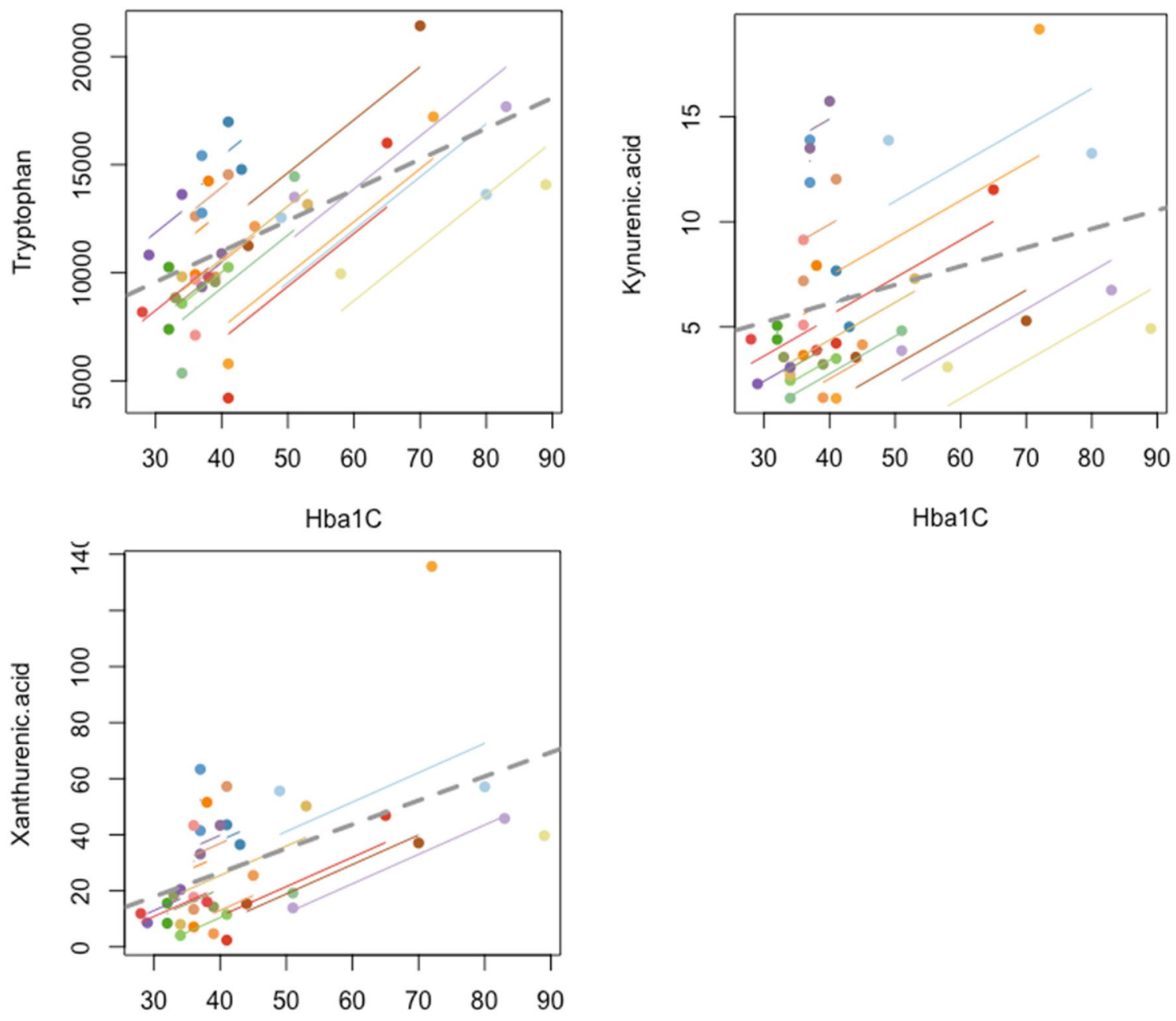

Hba1C

Figure 6. Repeated measure correlation plots of $\mathrm{HbAlc}$ with tryptophan, kynurenic acid and xanthurenic acid levels respectively.Metabolite units ( $\mathrm{ng} / \mathrm{ml}), \mathrm{HbAlc}(\mathrm{mmol} / \mathrm{mol})$.

Although the precise molecular mechanisms of XA role in T2D have not yet been elucidated, historical publications have proposed multiple mechanisms, including zinc chelation of XA leading to the formation of complexes that present identically to insulin antigens ${ }^{36-38}$, or the possibility that XA exhibits a direct toxic, and inhibitive effect on pancreatic islets ${ }^{38-40}$. The role of XA in T2D resolution post bariatric surgery warrants further investigation, as modulation of this pathway may be a target for future intervention in individuals with pre-surgical T2D.

In addition to KMO, the enzymes KYNU and KAT may also play a role in the relationship between the kynurenine pathway, obesity and T2D. For example, vitamin B6 is vital co-factor for both enzymes ${ }^{41}$, deficiency of which is also associated with obesity ${ }^{42}$ and chronic inflammation ${ }^{41}$. KYNU is more sensitive to vitamin B6 deficiency compared to KAT and thus KYNU activity is downregulated in instances of deficiency. This causes a pathway shift leading to increased production of XA and $\mathrm{KYNA}^{43}$, which is consistent with our data, where we observed higher concentrations of XA and KYNA in the pre-operative groups, where BMI and therefore levels of obesity were more extreme (Tables 3, 4). Furthermore, the reduction in BMI after surgery was also associated with reduced concentrations of these metabolites and improved glycaemic control, which potentially is due to the reduction in systemic inflammation.

The impact of bariatric surgery on serum KYN metabolites may also have implications beyond glycaemic control. Elevated kynurenic acid levels have been associated with an increased risk of acute coronary events in individuals with chronic heart disease $\mathrm{e}^{44}$. In one study, tryptophan metabolite levels were shown to be positively correlated with severity of chronic kidney disease with a direct nephrotoxic effect hypothesised ${ }^{45}$. Improvement in renal function after bariatric surgery is now becoming more evident ${ }^{46}$, and regulation of tryptophan metabolites may also be partly responsible for improvement in such conditions.

There are also links between the TRP pathway and the central nervous system. Cytokine mediated inflammation has been implicated in dysregulation of the hypothalamus-pituitary-adrenal axis and can lead to depression ${ }^{47}$. Kynurenic acid and quinolinic acid are both of interest in neurogastroenterology. Both metabolites are neuroactive, with KYNA acting as a NMDA antagonist and QA considered to be a NMDA agonist ${ }^{48}$. These 
metabolites can influence neuronal activity in both the central and peripheral nervous systems, with the former being neuroprotective and the latter neurotoxic. From a bariatric surgery perspective, the key to relationship between tryptophan metabolism and the CNS may lie in the gut microbiome. The concept of the gut-brain axis is becoming more established ${ }^{49}$ and the gut microbiota are now thought to influence brain, mood and cognitive function. An example is found in patients with irritable bowel syndrome where TRP-KYN pathway metabolites kynurenic acid and serotonin levels measured in small bowel mucosa were found to be directly correlated with anxiety and depression scores. Furthermore, chronic low tryptophan states also appear to accelerate the conversion of serotonin to 5 hydroxyindoleacetic acid ${ }^{50}$. This growing body of research has led to the proposal of the hypothesis that changes in GI tract metabolism, associated with the TRP-KYN pathway, may influence psychological states ${ }^{51}$. As bariatric surgery reduces serum tryptophan, kynurenines and other macronutrient levels, it remains to be seen as to whether this effect may have an impact on the increased incidence of depression after bariatric surgery ${ }^{52}$ and warrants further investigation.

Finally, the actual bioavailability of tryptophan and therefore its downstream metabolites are reported to be directly influenced by the gut microbiome ${ }^{49}$. Gut microbes have been reported to metabolise tryptophan to indole species, which in turn play a mechanistic role in microbiota to host signalling ${ }^{25,48}$. Indoles, including IAA, that are produced from microbial metabolism of tryptophan are thought to act on the aryl hydrocarbon receptor (AhR) in humans and influence gut barrier function ${ }^{53}$. This relationship is becoming a focus of research in obesity ${ }^{15}$ and GI permeability post bariatric surgery ${ }^{54}$. In our data, there was no significant change in IAA concentrations in the pre- and post- surgery comparisons, indicating that gut-microbial metabolism of tryptophan was not an influencing contributing factor in the phenotypic changes observed. Further investigations into additional gut microbially derived indoles would be of benefit and would facilitate further exploration of the gut microbiome's influence on metabolism post bariatric surgery, however measurement of an additional metabolic pathway was beyond the scope of this study.

Limitations. While longitudinal, this study only investigated the immediate metabolic changes 3 months after surgery. As a discovery study, the relatively small sample size limits the generalisability of the results and may decrease the power of the study. However, the widespread physiological and metabolic effects exerted by bariatric surgery means inter-individual variability is small compared to the overarching effects of the surgery $y^{55}$. The paired nature of this study also permitted us to measure and compare the differences in response between individuals as well as the surgical procedure. It will be important to evaluate the longer-term changes, ideally with further comparison groups of cohorts without obesity in order to validate the results.

There was an imbalance in the gender distribution of study participants (F:M, 15:5) which also needs to be considered: this however, was in keeping with the 3:1 (F:M) ratio of patients undergoing primary bariatric surgery in the United Kingdom ${ }^{56}$. Other factors that influence the TRP-KYN pathway such as vitamin B levels and the other derivatives of tryptophan such as the indole and its derivatives also merit investigation.

Finally, it should be considered that there is the potential that a reduction in overall nutrient intake postsurgery is driving the observations. A subset of 11 patients provided dietary information in the form of a recall online questionnaire (see Supplementary 5). General reductions in total calories consumed and all macronutrients were significant at 3-months post-operative. In particular, a reduction in average pre-operative protein intake of $80.6 \mathrm{~g} /$ day compared to $49.7 \mathrm{~g} /$ day post-operatively $(\mathrm{p}=0.014)$ was reported. This is consistent with previous studies ${ }^{57}$ and could explain the reduction in tryptophan bioavailability. However, if dietary influence was the only factor at play in these observations, it would be expected that all metabolites in the pathway would be significantly reduced post-surgery, which was not the case, indicating the perturbations observed in the current study were associated with specific enzymatic steps of the pathway. A separate controlled study dedicated to investigating the effects of diet on the TRP-KYN pathway metabolites and its effects on T2D would be of interest as future work.

\section{Conclusion}

Bariatric surgery, in particular RYGB reduces serum levels of tryptophan and its downstream kynurenine metabolites including kynurenic acid and xanthurenic acid. These metabolites are associated with T2D and its reduction after surgery correlates with a reduction in HbA1c levels and BMI. Whether this is a cause or consequence remains to be further established. The reduction of these metabolites may also have a wide range of beneficial and unintended effects beyond weight loss and glycaemic control.

Received: 28 May 2021; Accepted: 3 December 2021

Published online: 07 January 2022

\section{References}

1. Schauer, P. R. et al. Bariatric surgery versus intensive medical therapy for diabetes-5-year outcomes. N. Engl. J. Med. 376(7), 641-651 (2017).

2. Cummings, D. E. \& Rubino, F. Metabolic surgery for the treatment of type 2 diabetes in obese individuals. Diabetologia $61(2)$, 257-264 (2018).

3. Wiggins, T., Guidozzi, N., Welbourn, R., Ahmed, A. R. \& Markar, S. R. Association of bariatric surgery with all-cause mortality and incidence of obesity-related disease at a population level: A systematic review and meta-analysis. PLoS Med. 17(7), e1003206 (2020)

4. Hofsø, D. et al. Gastric bypass versus sleeve gastrectomy in patients with type 2 diabetes (Oseberg): A single-centre, triple-blind, randomised controlled trial. Lancet Diabetes Endocrinol. 7(12), 912-924 (2019).

5. O'Brien, R. et al. Microvascular outcomes in patients with diabetes after bariatric surgery versus usual care: A matched cohort study. Ann. Intern. Med. 169(5), 300-310 (2018). 
6. Batterham, R. L. \& Cummings, D. E. Mechanisms of diabetes improvement following bariatric/metabolic surgery. Diabetes Care 39(6), 893-901 (2016).

7. Laferrère, B. \& Pattou, F. Weight-independent mechanisms of glucose control after Roux-en-Y gastric bypass. Front. Endocrinol. 9, 530 (2018).

8. Sharples, A. J. \& Mahawar, K. Systematic review and meta-analysis of randomised controlled trials comparing long-term outcomes of Roux-En-Y gastric bypass and sleeve gastrectomy. Obes. Surg. 30(2), 664-672 (2020).

9. Zhang, Y. et al. Improvement in nocturnal hypoxemia in obese patients with obstructive sleep apnea after bariatric surgery: A meta-analysis. Obes. Surg. 29(2), 601-608 (2019).

10. McTigue, K. M. et al. Comparing the 5-year diabetes outcomes of sleeve gastrectomy and gastric bypass: The National PatientCentered Clinical Research Network (PCORNet) bariatric study. JAMA Surg. 155, e200087 (2020).

11. Wallenius, V. et al. Sleeve gastrectomy and Roux-en-Y gastric bypass in the treatment of type 2 diabetes. Two-year results from a Swedish multicenter randomized controlled trial. Surg. Obes. Relat. Dis. 16, 1035 (2020).

12. Aminian, A. Bariatric procedure selection in patients with type 2 diabetes: Choice between Roux-en-Y gastric bypass or sleeve gastrectomy. Surg. Obes. Relat. Dis. 16(2), 332-339 (2020).

13. Wensveen, F. M. et al. NK cells link obesity-induced adipose stress to inflammation and insulin resistance. Nat. Immunol. 16(4), 376-385 (2015).

14. Ahmad, M. S. et al. Metabolic phenotype of obesity in a Saudi population. J. Proteome Res. 16(2), 635-644 (2017).

15. Cussotto, S. et al. Tryptophan metabolic pathways are altered in obesity and are associated with systemic inflammation. Front. Immunol. 11, 557 (2020).

16. Richard, D. M. et al. L-tryptophan: Basic metabolic functions, behavioral research and therapeutic indications. Int. J. Tryptophan. Res. 2, 45-60 (2009).

17. Cervenka, I., Agudelo, L. Z. \& Ruas, J. L. Kynurenines: Tryptophan's metabolites in exercise, inflammation, and mental health. Science (New York). 357, 6349 (2017).

18. Badawy, A.A.-B. Tryptophan metabolism: A versatile area providing multiple targets for pharmacological intervention. Egypt J. Basic Clin. Pharmacol. https://doi.org/10.32527/2019/101415 (2019).

19. Oxenkrug, G. F. Metabolic syndrome, age-associated neuroendocrine disorders, and dysregulation of tryptophan-kynurenine metabolism. Ann. N. Y. Acad. Sci. 1199, 1-14 (2010).

20. Schröcksnadel, K., Wirleitner, B., Winkler, C. \& Fuchs, D. Monitoring tryptophan metabolism in chronic immune activation. Clin. Chim. Acta Int. J. Clin. Chem. 364(1-2), 82-90 (2006).

21. Oxenkrug, G. Insulin resistance and dysregulation of tryptophan-kynurenine and kynurenine-nicotinamide adenine dinucleotide metabolic pathways. Mol. Neurobiol. 48(2), 294-301 (2013).

22. NICE. National Institute for Health and Care Excellence-Obesity: Identification, Assessment and Management (NICE Guideline 189) (NICE, 2014).

23. Whiley, L. et al. Ultrahigh-performance liquid chromatography tandem mass spectrometry with electrospray ionization quantification of tryptophan metabolites and markers of gut health in serum and plasma-application to clinical and epidemiology cohorts. Anal. Chem. 91(8), 5207-5216 (2019).

24. Crenn, P., Messing, B. \& Cynober, L. Citrulline as a biomarker of intestinal failure due to enterocyte mass reduction. Clin. Nutr. 27(3), 328-339 (2008).

25. Hubbard, T. D., Murray, I. A. \& Perdew, G. H. Indole and tryptophan metabolism: Endogenous and dietary routes to ah receptor activation. Drug Metab. Dispos. 43(10), 1522-1535 (2015).

26. Benjamini, Y. \& Hochberg, Y. Controlling the false discovery rate: A practical and powerful approach to multiple testing. J. R. Stat. Soc. Ser. B (Methodol.) 57(1), 289-300 (1995).

27. Bakdash, J. Z. \& Marusich, L. R. Repeated measures correlation. Front. Psychol. https://doi.org/10.3389/fpsyg.2017.00456 (2017).

28. Bland, J. M. \& Altman, D. G. Statistics notes: Calculating correlation coefficients with repeated observations: Part 1-Correlation within subjects. BMJ (Clin. Res. Ed.) 310(6977), 446 (1995).

29. Bland, J. M. \& Altman, D. G. Calculating correlation coefficients with repeated observations: Part 2-Correlation between subjects. BMJ (Clin. Res. Ed.) 310(6980), 633 (1995).

30. Gürcü, S. et al. Neopterin and biopterin levels and tryptophan degradation in patients with diabetes. Sci. Rep. 10(1), 17025 (2020).

31. Favennec, M. et al. The kynurenine pathway is activated in human obesity and shifted toward kynurenine monooxygenase activation. Obesity (Silver Spring) 23(10), 2066-2074 (2015).

32. Rosen, D. A., Maengwyn-Davies, G. D., Becker, B., Stone, H. H. \& Friedenwald, J. S. Xanthurenic acid excretion studies in diabetics with and without retinopathy. Proc. Soc. Exp. Biol. Med. 88(3), 321-323 (1955).

33. Oh, J. S. et al. Urinary profiling of tryptophan and its related metabolites in patients with metabolic syndrome by liquid chromatography-electrospray ionization/mass spectrometry. Anal. Bioanal. Chem. 409(23), 5501-5512 (2017).

34. Oxenkrug, G. F. Increased plasma levels of xanthurenic and kynurenic acids in type 2 diabetes. Mol. Neurobiol. 52(2), 805-810 (2015).

35. Favennec, M. et al. Post-bariatric surgery changes in quinolinic and xanthurenic acid concentrations are associated with glucose homeostasis. PLoS ONE 11(6), e0158051 (2016).

36. Kotake, Y. Xanthurenic acid, an abnormal metabolite of tryptophan and the diabetic symptoms caused in albino rats by its production. J. Vitaminol. (Kyoto) 1(2), 73-87 (1955).

37. Kotake, Y., Ueda, T., Mori, T., Igaki, S. \& Hattori, M. Abnormal tryptophan metabolism and experimental diabetes by xanthurenic acid (XA). Acta Vitaminol. Enzymol. 29(1-6), 236-239 (1975).

38. Meyramov, G., Korchin, V. \& Kocheryzkina, N. Diabetogenic activity of xanturenic acid determined by its chelating properties? Transpl. Proc. 30(6), 2682-2684 (1998).

39. Malina, H. Z., Richter, C., Mehl, M. \& Hess, O. M. Pathological apoptosis by xanthurenic acid, a tryptophan metabolite: Activation of cell caspases but not cytoskeleton breakdown. BMC Physiol. 1, 7 (2001).

40. Rogers, K. S. \& Evangelista, S. J. 3-Hydroxykynurenine, 3-hydroxyanthranilic acid, and o-aminophenol inhibit leucine-stimulated insulin release from rat pancreatic islets. Proc. Soc. Exp. Biol. Med. 178(2), 275-278 (1985).

41. Ueland, P. M., McCann, A., Midttun, Ø. \& Ulvik, A. Inflammation, vitamin B6 and related pathways. Mol. Aspects Med. 53, 10-27 (2017).

42. Aasheim, E. T., Hofsø, D., Hjelmesaeth, J., Birkeland, K. I. \& Bøhmer, T. Vitamin status in morbidly obese patients: A cross-sectional study. Am. J. Clin. Nutr. 87(2), 362-369 (2008).

43. Mascolo, E. \& Vernì, F. Vitamin B6 and diabetes: Relationship and molecular mechanisms. Int. J. Mol. Sci. 21(10), 3669 (2020).

44. Eussen, S. J. et al. Kynurenines as predictors of acute coronary events in the Hordaland Health Study. Int. J. Cardiol. 189, 18-24 (2015).

45. Debnath, S. et al. Tryptophan metabolism in patients with chronic kidney disease secondary to type 2 diabetes: Relationship to inflammatory markers. Int. J. Tryptophan. Res. 10, 1178646917694600 (2017).

46. Docherty, N. G. \& Le Roux, C. W. Bariatric surgery for the treatment of chronic kidney disease in obesity and type 2 diabetes mellitus. Nat. Rev. Nephrol. 16, 709 (2020).

47. Moulton, C. D., Pickup, J. C. \& Ismail, K. The link between depression and diabetes: The search for shared mechanisms. Lancet Diabetes Endocrinol. 3(6), 461-471 (2015). 
48. Kennedy, P. J., Cryan, J. F., Dinan, T. G. \& Clarke, G. Kynurenine pathway metabolism and the microbiota-gut-brain axis. Neuropharmacology $112(\mathrm{Pt} \mathrm{B}), 399-412$ (2017).

49. O’Mahony, S. M., Clarke, G., Borre, Y. E., Dinan, T. G. \& Cryan, J. F. Serotonin, tryptophan metabolism and the brain-gutmicrobiome axis. Behav. Brain Res. 277, 32-48 (2015).

50. Matsuoka, K. et al. Concentrations of various tryptophan metabolites are higher in patients with diabetes mellitus than in healthy aged male adults. Diabetol. Int. 8(1), 69-75 (2017).

51. Keszthelyi, D. et al. Decreased levels of kynurenic acid in the intestinal mucosa of IBS patients: Relation to serotonin and psychological state. J. Psychosom. Res. 74(6), 501-504 (2013).

52. Lu, C. W. et al. Increased risk for major depressive disorder in severely obese patients after bariatric surgery-A 12-year nationwide cohort study. Ann. Med. 50(7), 605-612 (2018).

53. Scott, S. A., Fu, J. \& Chang, P. V. Microbial tryptophan metabolites regulate gut barrier function via the aryl hydrocarbon receptor. Proc. Natl. Acad. Sci. 117(32), 19376 (2020).

54. Jennis, M. et al. Microbiota-derived tryptophan indoles increase after gastric bypass surgery and reduce intestinal permeability in vitro and in vivo. Neurogastroenterol. Motil. 30(2), e13178 (2018).

55. Li, J. V. et al. Roux-en-Y gastric bypass-induced bacterial perturbation contributes to altered host-bacterial co-metabolic phenotype. Microbiome 9(1), 139 (2021).

56. Society B-BOMS. NBSR Third Registry Report (2020).

57. Raatz, S. K. et al. Reported nutrient intake over 7 years after Roux-en-Y gastric bypass in the longitudinal assessment of bariatric surgery-3 (LABS-3) psychosocial study. Surg. Obes. Relat. Dis. 16(8), 1022-1029 (2020).

\section{Acknowledgements}

Funding for this project was provided by an Imperial College Healthcare NHS Trust-Biomedical Research Centre (BRC) Surgery \& Surgical Technology Pilot Project 2019-2020 Grant awarded to KTDY. Funding was also provided by the Diabetes Research and Wellness Foundation through the Sutherland-Earl Clinical Research Fellowship 2015 awarded to NP. This work was also supported by the Medical Research Council and National Institute for Health Research Grant Number: MC_PC_12025. Infrastructure support was provided by the National Institute for Health Research (NIHR) Imperial BRC.

\section{Author contributions}

The manuscript idea conceived by all authors. N.P. and K.T.D.Y. collected specimens for analysis. The data were acquired at the National Phenome Centre where ML is COO. K.T.D.Y. performed the data analysis. Data analytic support provided by L.W., M.L. and E.H. K.T.D.Y. lead in writing the manuscript with analytical and interpretation support from all authors. S.P., A.D. and E.H. supervised the project. All authors discussed the results and contributed to the final manuscript.

\section{Competing interests}

The authors declare no competing interests.

\section{Additional information}

Supplementary Information The online version contains supplementary material available at https://doi.org/ 10.1038/s41598-021-03833-4.

Correspondence and requests for materials should be addressed to E.H.

Reprints and permissions information is available at www.nature.com/reprints.

Publisher's note Springer Nature remains neutral with regard to jurisdictional claims in published maps and institutional affiliations.

(c) Open Access This article is licensed under a Creative Commons Attribution 4.0 International

License, which permits use, sharing, adaptation, distribution and reproduction in any medium or format, as long as you give appropriate credit to the original author(s) and the source, provide a link to the Creative Commons licence, and indicate if changes were made. The images or other third party material in this article are included in the article's Creative Commons licence, unless indicated otherwise in a credit line to the material. If material is not included in the article's Creative Commons licence and your intended use is not permitted by statutory regulation or exceeds the permitted use, you will need to obtain permission directly from the copyright holder. To view a copy of this licence, visit http://creativecommons.org/licenses/by/4.0/.

(c) The Author(s) 2022 